\title{
Hiding beyond plain sight: Textural analysis of positron emission tomography to identify high-risk plaques in carotid atherosclerosis
}

\author{
Manish Motwani, MB ChB, PhD, FSCMR, FSCCT ${ }^{\mathrm{a}, \mathrm{b}}$ \\ a Department of Cardiology, Manchester Heart Centre, Manchester Royal Infirmary, Manchester \\ University NHS Foundation Trust, Manchester, UK \\ b Institute of Cardiovascular Science, University of Manchester, Manchester, UK
}

Received Nov 26, 2019; accepted Nov 26, 2019

doi: 10.1007/s12350-019-01981-9

\section{See related article, pp. $1861-1871$}

Over the last few decades, technological advances in hardware and acquisition techniques have produced ever more complex and sophisticated data-rich medical images. By comparison, the interpretation of these images has remained largely dependent on manual processing by expert humans with more recently variable levels of operator-driven software assistance. The latter has proven to be a dependable and successful model. Arguably, nothing need change, but on the other hand, if we accept that there may be additional clues beneath the surface and beyond our human perception in increasingly data-rich images, it would be against our pioneering spirit as medical investigators to sit complacently and not explore methods of extracting that hidden data.

Currently, most medical imaging is assessed visually, with substantial amounts of imperceptible but feature-rich information unwittingly discarded. Medical images from most modalities using modern scanner hardware typically contain between 12 and 16 bits/pixel, which corresponds to 4,096-65,536 shades of gray. ${ }^{1}$ However, even on medical-grade displays, we have a much lower number of gray shades available to review (typically up to 1,024); and it is estimated that the human eye can perceive no more than around 900

Reprint requests: Manish Motwani, MB ChB, PhD, FSCMR, FSCCT, Department of Cardiology, Manchester Heart Centre, Manchester Royal Infirmary, Manchester University NHS Foundation Trust, Oxford Road, Manchester, UK; Mani.Motwani@mft.nhs.uk J Nucl Cardiol 2021;28:1872-4.

$1071-3581 / \$ 34.00$

Copyright (c) 2019 American Society of Nuclear Cardiology. shades of gray anyway. ${ }^{1}$ So, for as long as image analysis remains a human enterprise, it is subject to the limitations of human ability and the extraction of additional features to finesse interpretation requires a more technologically advanced approach. One potential way of accessing this hidden information is by using radiomics, which is the mathematical extraction of large amounts of quantitative features from clinical imaging using data characterization algorithms. ${ }^{2}$

Texture analysis is one form of radiomic assessment that specifically assesses the variation in grayscale image intensities within an image, i.e., radiologic texture. ${ }^{3}$ The underlying assumption in texture analysis is that grayscale values and their spatial and temporal interrelationships within an image reflect phenotypic variations in the imaged tissue and may indicate molecular variations. ${ }^{3,4}$ Recently, there has been a substantial interest in texture analysis in the oncology setting. Several studies have utilized texture analysis of Fluorine-18 fluorodeoxyglucose positron emission tomography (18F-FDG PET) to assess intra-tumoral heterogeneity which is associated with tumor aggressiveness $^{5,6}$; and have shown promising results in predicting treatment response and patient survival. ${ }^{7-12}$

By comparison, there has been only limited study of texture analysis techniques in vascular PET imaging and atherosclerosis. However, in this issue of the Journal of Nuclear Cardiology, Kafouris et al assess the feasibility of texture analysis in carotid arteries using 18F-FDG PET-CT images to predict histological and immunohistochemical features of event-prone plaque. ${ }^{13}$ Sixtyseven different 18F-FDG PET-based textural features were extracted from carotid images of 21 patients with high-grade carotid stenosis undergoing endarterectomy. Accepting the acknowledged limitations including a small sample size and a considerable spectrum bias, the authors found several textural-based metrics showing 
significant promise in independently identifying confirmed histopathological features of plaque vulnerability and in some cases with incremental accuracy over standard target-to-background ratio (TBR) assessments.

Regarding the concept of identifying carotid artery event-prone plaque more broadly, some cautions are worth noting from parallel studies of coronary artery disease $(\mathrm{CAD})$. In the latter, the search for the vulnerable plaque has been extensive and resource-intensive, and so far, has also failed to change clinical care. ${ }^{14}$ Although retrospective studies have successfully linked culprit plaque to different adverse imaging characteristics, prospective studies have suggested that these supposedly high-risk plaques rarely go on to cause clinical events themselves. ${ }^{15}$ The likely explanation for this apparent discrepancy is that the majority of 'highrisk' plaques actually heal without rupturing. Intuitively, these same limitations may also apply to carotid artery atherosclerosis. ${ }^{16}$ However, combining multiple adverse plaque characteristics does seems to improve the positive predictive value of events in $\mathrm{CAD}$, and possibly this supports attempts at more sophisticated analysis such as texture analysis. ${ }^{14,15}$ Whether such approaches in coronary or carotid atherosclerosis will ever be sufficient to prompt plaque-specific revascularization or aggressive systemic therapies is, however, the subject of considerable debate ${ }^{14,17}$

Leaving aside the occasional hysteria of artificial intelligence replacing all radiologists, the principle of radiomics tackles a more fundamental recognition that there is a biological limitation to human capability. ${ }^{18}$ Until perhaps human evolution catches up-and incidentally there are already a small number of women called tetrachromats possessing a mutation allowing them to perceive 100 million rather than just 1 million colors-radiomics provides us with more information than comprehensible by visual inspection with each lesion being characterized by hundreds of different parameters. $^{19}$

Finally, although visual inspection is sufficient for clinical diagnosis, there is a growing need for quantitative image analysis techniques that can further increase our understanding of disease processes and the precision of image-based risk prediction. Radiologic examinations are vast spatial data sets, where every voxel is a potential data mine itself, and radiomics tries to utilize this information by quantifying properties beyond plain human sight. However, clinical translation is still a challenge as we currently lack adequate large-scale evidence to support the scientific claims-but with ongoing development the potential for this emerging field is bewildering.

\section{References}

1. Kimpe $\mathrm{T}$, Tuytschaever $\mathrm{T}$. Increasing the number of gray shades in medical display systems- How much is enough? J Digit Imaging 2007;20(4):422-32. https://doi.org/10.1007/s10278-006-1052-3.

2. Gillies RJ, Kinahan PE, Hricak H. Radiomics: Images are more than pictures, they are data. Radiology 2016;278:563-77.

3. Varghese BA, Steven Y. Cen SY, Hwang DH, Duddalwar VA. Texture analysis of imaging: What radiologists need to know. AJR Am J Roentgenol 2019;212(3):520-528

4. Aerts HJ, Velazquez ER, Leijenaar RT, Parmar C, Grossmann P, Carvalho $\mathrm{S}$, et al. Decoding tumour phenotype by noninvasive imaging using a quantitative radiomics approach. Nat Commun 2014;5:4006.

5. Gay L, Baker AM, Graham TA. Tumour cell heterogeneity. F1000Res 2016; 5:238

6. Cyll K, Ersvær E, Vlatkovic L, Pradhan M, Kildal W, Avranden Kjær M, et al. Tumour heterogeneity poses a significant challenge to cancer biomarker research. Br J Cancer 2017;117:367.

7. Chicklore S, Goh V, Siddique M, Roy A, Marsden PK, Cook GJ. Quantifying tumour heterogeneity in 18F-FDG PET/CT imaging by texture analysis. Eur J Nucl Med Mol Imaging 2013;40:133-40.

8. Hatt M, Tixier F, Pierce L, Kinahan PE, Le Rest CC, Visvikis D. Characterization of PET/CT images using texture analysis: The past, the present....any future? Eur J Nucl Med Mol Imaging 2017;44:151-65.

9. Tixier F, Le Rest CC, Hatt M, Albarghach N, Pradier O, Metges JP, et al. Intratumor heterogeneity characterized by textural features on baseline 18F-FDG PET images predicts response to concomitant radiochemotherapy in esophageal cancer. J Nucl Med 2011;52:369-78.

10. Hatt M, Tixier F, Cheze-le Rest C, Pradier O, Visvikis D. Robustness of intratumour 18F-FDG PET uptake heterogeneity quantification for therapy response prediction in oesophageal carcinoma. Eur J Nucl Med Mol Imaging 2013;40:1662-71.

11. El Naqa I, Grigsby P, Apte A, Kidd E, Donnelly E, Khullar D, et al. Exploring feature-based approaches in PET images for predicting cancer treatment outcomes. Pattern Recognit 2009;42:1162-71.

12. Tixier F, Hatt M, Le Rest CC, Le Pogam A, Corcos L, Visvikis D. Reproducibility of tumor uptake heterogeneity characterization through textural feature analysis in 18F-FDG PET. J Nucl Med 2012;53:693-700.

13. Kafouris PP, Koutagiar IP, Georgakopoulos AT, Spyrou GM, Visvikis D, Anagnostopoulos CD. Fluorine-18 fluorodeoxyglucose positron emission tomography-based textural features for prediction of event prone carotid atherosclerotic plaques. J Nucl Cardiol 2019. https://doi.org/10.1007/s12350-019-01943-1.

14. Dweck M, Doris M, Motwani M, et al. Imaging of coronary atherosclerosis-Evolution towards new treatment strategies. Nat Rev Cardiol 2016;13:533-48.

15. Stone GW, et al. A prospective natural-history study of coronary atherosclerosis. N Engl J Med 2011;364:226-35.

16. Mann J, Davies MJ. Mechanisms of progression in native coronary artery disease: Role of healed plaque disruption. Heart 1999;82:265-8.

17. Arbab-Zadeh A, Fuster V. The myth of the 'vulnerable plaque': Transitioning from a focus on individual lesions to atherosclerotic disease burden for coronary artery disease risk assessment. J Am Coll Cardiol 2015;65:846-55.

18. Langlotz, CP. Will artificial intelligence replace radiologists? Radiol 2019 1(3):e190058 
19. Kolossvary M, Kellermayer M, Merkely B, Maurovich-Horvat P. Cardiac computed tomography radiomics: A comprehensive review on radiomic techniques. J Thorac Imaging 2018;33:26-34.
Publisher's Note Springer Nature remains neutral with regard to jurisdictional claims in published maps and institutional affiliations. 